\title{
Laboratory Test Results Anatomical Region of Specimen
}

National Cancer Institute

\section{Source}

National Cancer Institute. Laboratory Test Results Anatomical Region of Specimen. NCI

Thesaurus. Code C119844.

The specific anatomical or biological region within the tissue or organ specimen used in the laboratory assessment. 